In 1952, Wilson coined the currently preferred term necrotising fasciitis' to emphasise the constant feature of necrotic fascia with spread of infection along fascial planes and non-specificity of the bacterial aetiology. ${ }^{4}$

Necrotising fasciitis commonly occurs in the lower extremities, trunk and perineum but rarely involves the face. There is often, though not always, an inciting cause such as a laceration or an infection. Patients frequently have an underlying predisposing condition such as diabetes or alcoholism. ${ }^{4}$ Group A and non-group A Streptococcus and Staphylococcus species are the most common aetiological agents but a mixture of facultative and anaerobic organisms can also lead to this type of infection. ${ }^{2,3}$

The diagnosis depends on clinical awareness and a high index of suspicion about the condition. The signs and symptoms can be nondescript initially but pain is usually out of proportion to clinical signs and patients are significantly unwell. ${ }^{5}$ It is important to commence appropriate antibiotics intravenously once the condition is diagnosed clinically. Samples for bacteriological studies should be obtained before institution of antibiotic therapy. This should include Gram stains of the discharge and aerobic and anaerobic cultures of the wound and blood.

Antibiotic therapy, in particular high-dose intravenous penicillin, has proven efficacy in treating Group A streptococcal infection, ${ }^{6}$ but in some cases of multi-bacillary disease, as in necrotising fasciitis, there is a suboptimal response to penicillin alone. There is emerging evidence of the role of clindamycin in the treatment. ${ }^{7}$ As patients with necrotising fasciitis have toxaemia as a result of streptococcal exotoxins type A, clindamycin, which works by interfering with protein (toxin) production at the ribosomal level, may be helpful in managing the condition even before the organism is killed by beta-lactam antibiotics. ${ }^{8}$

The importance of surgical debridement has long been recognised. ${ }^{9}$ Debridement following involvement of the periorbital region can result in cosmetic disfigurement and functional problems such as corneal exposure. Reconstruction of the eyelids and skin grafts may be necessary to avoid complications such as cicatricial lid retraction and lagophthalmos.

The mortality in patients with periorbital spread is reported to be $12.5 \%{ }^{1}$ and necrotising fasciitis of the head and neck region has an overall mortality of between $9 \%$ and $31 \%$ in the antibiotic era. ${ }^{10}$ The prognosis is adversely affected by delay in diagnosis and extension of infection from the face to the neck.

The history was misleading in this case because of trauma, but the signs of necrotising fasciitis were unmistakable within $24 \mathrm{~h}$ of injury. It must be emphasised that diagnosis must be made clinically because late diagnosis increases the mortality. We seek to reiterate the importance of early treatment with highdose intravenous antibiotics, including penicillin, which in this case limited the extent of infection and reduced the need for extensive debridement and reconstruction.
1. Kronish JW, Mcleash MW. Eyelids necrosis and periorbital necrotising fasciitis. Ophthalmology 1991;98:92-8.

2. Shindo ML, Nalbone NP, Dougherty WR. Necrotising fasciitis of the face. Laryngoscope 1997;107:1071-9.

3. Giuliano A, Lewis F Jr, Hadley K, et al. Bacteriology of necrotizing fasciitis. Am J Surg 1997;134:52-7.

4. Wilson B. Necrotizing fasciitis. Am Surg 1952;18:416-31.

5. Haywood CT, McGear A, Lowe DE. Clinical experience with 20 cases of Group A streptococcal necrotizing fasciitis and myonecrosis. Plast Reconstr Surg 1999;5:1567-73.

6. Bisno AL, Stevens DL. Streptococcal infections of skin and soft tissues. N Engl J Med 1996;334:240.

7. Brun-Buisson C. The therapeutic approach to necrotizing fasciitis. Ann Dermatol Venereol 2001;128(3-C2):394-403.

8. Norby SR, Norrby-Teglund A. Infections due to group A streptococcus: new concepts and potential treatment. Ann Acad Med Singapore 1997;26:691-3.

9. Wood TF, Potter MA, Jonasson O. Streptococcal toxic shock like syndrome: the importance of surgical intervention. Ann Surg 1993;217:109.

10. Buchanan CS, Haserick JR. Necrotizing fasciitis due to group AB haemolytic streptococci. Arch Dermatol 1970;101:664-8.

B. Pal

S. Evans

R.F. Walters

Department of Ophthalmology

University Hospital of Wales

Cardiff, UK

B. Pal

Leeds General Infirmary

Clarendon Wing

Belmont Grove

Leeds LS2 9NS, UK

Tel: +44 (0)1132432799

Fax: +44 (0)1132926239

e-mail: bishpall7@aol.com

Sir,

\section{Conjunctival metastasis: a rare presentation of} bronchial adenocarcinoma

Metastatic involvement of ocular tissues in malignancy is not uncommon; however, tumour deposits in the conjunctiva are extremely rare and indeed few cases are documented in the literature. We report a patient presenting with a rapidly enlarging conjunctival mass found to be an adenocarcinomatous metastasis from an occult pulmonary primary.

\section{Case report}

A 68-year-old man presented with a 10-day history of mild irritation around his left medial canthus associated with a rapidly enlarging painless mass. There were no other complaints or relevant history. On direct questioning he admitted to a loss of appetite and significant weight loss over the preceding month.

Examination revealed the presence of a $18 \mathrm{~mm}$ by $13 \mathrm{~mm}$ solid mass arising from the nasal conjunctiva of the left eye (Fig. 1). The anterior segments were otherwise normal and dilated fundoscopy was unremarkable. Systemic examination revealed the presence of left-sided cervical and supraclavicular lymphadenopathy. 


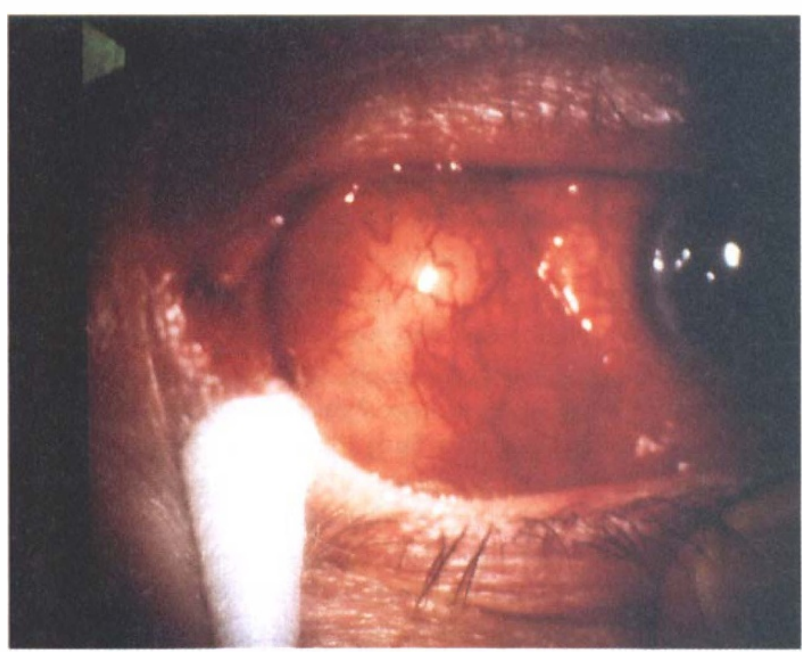

Fig. 1. Photograph of the left medial conjunctival mass $(\times 12)$.

A CT scan of the head revealed no evidence of sinus mass or orbital tumour extension. At excision biopsy, the lesion was found to be arising from the conjunctiva with a clear plane of separation from sclera. Histopathological examination revealed features of a malignant neoplasm with glandular, epithelial differentiation.

Referral was made to an oncologist and subsequent thoracoabdominal CT identified the presence of a left lower lobe bronchial adenocarcinoma with bilateral adrenal metastases. The patient declined chemotherapeutic intervention. Two months after initial presentation he complained of visual field loss. Clinical examination elicited a bitemporal hemianopia and brain $\mathrm{CT}$ revealed the presence of a chiasmal metastasis not seen previously (Fig. 2). The patient died 1 month later, 3 months after initial presentation, from disseminated carcinomatosis.

\section{Comment}

Metastatic carcinoma of the conjunctiva is a rare entity, emphasised by its sparse documentation in the literature.

Ocular metastasis from cutaneous melanoma is well documented and usually occurs to the uveal tract; however, conjunctival metastases from skin melanomas have previously been described. ${ }^{1,2}$

Spread to the conjunctiva from other non-cutaneous primary sites is less common. Metastasis of bowel adenocarcinoma to lid conjunctiva was described in 1957 by Ostriker. ${ }^{3}$ Kiratli and colleagues ${ }^{4}$ documented 10 cases of metastatic tumours to the conjunctiva. In these patients, the appearance of the conjunctival metastasis occurred in the advanced stage of disease and in the majority there were concurrent non-ocular metastases. At the time of diagnosis all the patients had a pre-existing history of primary or metastatic cancer.

In our case the patient's presenting symptom was the conjunctival mass and the diagnosis of metastatic malignancy was made without the presence of a known primary tumour. To our knowledge only one previous case has been reported where the presence of the conjunctival metastasis alerted the clinician to a distant silent primary. ${ }^{5}$

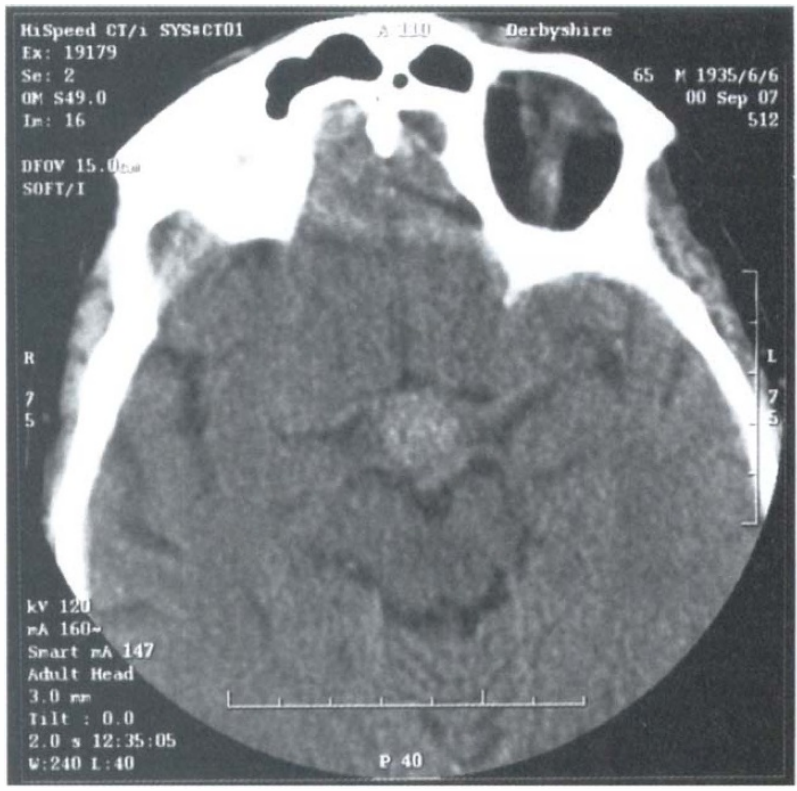

Fig. 2 Contrast-enhanced brain CT scan showing a mass in the region of the optic chiasm consistent with a chiasmal malignant metastasis.

The presence of a conjunctival metastasis should raise a high index of suspicion for other, non-ocular metastases. Once the primary tumour has been identified local treatment of the conjunctival mass may be considered by either external beam radiotherapy or excision if possible. Unfortunately the prognosis for patients with conjunctival metastasis is extremely poor due to the advanced underlying malignancy and its associated spread. ${ }^{4}$

\section{References}

1. Kwapiszeski BR, Savitt ML. Conjunctival metastasis from cutaneous melanoma as the initial sign of dissemination. Am J Ophthalmol 1997;123:266-8.

2. Jakobiec FA, Buckman G, Zimmerman LE, et al. Metastatic melonoma within and to the conjunctiva. Ophthalmology 1989;96:999-1005.

3. Ostriker PJ. Metastasis of adenocarcinoma of colon to the conjunctival surface of the lid. Arch Ophthalmol 1957;57:279-81.

4. Kiratli H, Shields CL, Shields JA, DePotter P. Metastatic tumours to the conjunctiva: report of 10 cases. $\mathrm{Br} \mathrm{J}$ Ophthalmol 1996;80:50-8.

5. Kuchle M, Holbach L, Schlotzer-Schrenhardt U. Gastric adenocarcinoma presenting as an eyelid and conjunctival mass. Eur J Ophthalmol 1992;2:3-9.

A. Alwitry

A.C. Browning

R. Holden

A. Joseph

Department of Ophthalmology

Derbyshire Royal Infirmary

Derby, UK

Mr A. Alwitry, MRCS

Department of Ophthalmology Derbyshire Royal Infirmary

London Road

Derby DE1 2QY, UK

Tel: +44 (0)1332 347141

Fax: +44 (0)1332 254677

e-mail: DocAmar@aol:com 